\title{
Alternative Approach of Inventive Problem Solving for Location Based Service Implementation
}

\author{
Song-Kyoo Kim, Senior Member, IACSIT
}

\begin{abstract}
Location Based Services and its applications such as Point of Interest and navigation are common applications for mobile phones. The applications are embedded even on low tier mobile devices. The paper deals another practical TRIZ adaptations for information technologies that is similar with the previous adaptation [2]. TRIZ (Teoriya Resheniya Izobretatelskikh Zadatch) that is also called TIPS (Theory of Inventive Problem Solving) is a methodology that is the practical innovation tool and the model-based technique for generating innovative ideas by Genrich Altshuller [1]. The key idea of the enhanced user experience is behavior analysis based on the typical statistical data that can be gathering during the regular user operations. This new user interface enhancing technology is designed to give simpler user experiences for the LBS applications by using TRIZ method.
\end{abstract}

Index Terms-Behavior analysis, Innovation, Location based services, TRIZ, TIPS, User experience.

\section{INTRODUCTION}

User experiences on consumer electronics such as a MP3 player, LBS applications on a mobile phone are considered as the target to apply the TRIZ for enhancement because current consumer electronics are getting complicated and hard to learn the user interfaces. Specially, enhancing the user experience for LBS applications by simplify the complexity is considered in the paper.

Currently, consumer electronics are powerful enough to support for improving the life style. Even the low tier device can support LBS applications. There are several ways to improve the determination of the initial position but all of methods are required additional process powers and/or radio frequencies. Human behavior in behavioral economics $[5,10]$ is considered for innovative user experience enhancement for LBS applications. Behavioral economics is a separate branches of economics that is using social, cognitive and emotional factors in understanding the economic decisions of consumers. Even though the topic is relative new area in economics, it is popular subject and has been announced to public as a famous best seller authored by Thaler [5].

The concept of the enhanced user experience for calculating the initial location position is behavior analysis based on the typical statistical data that can be gathering during the regular user operations. Even though they have the strength in different consumer electronics fields, both of companies keep one thing in their mind that is simplicities

Manuscript accepted June 13, 2011; revised July 1, 2011

Song-Kyoo Kim (amang.kim@samsung.com) is a technical manager at mobile communication division in Samsung Electronics, 94-1 Imsoo-Dong, Gumi, Kyungpook 730-350, South Korea (Tel:+82-54-479-3970; f:+82-54-479-5922) with giving users the high performed features. The objects that have mentioned are common contradiction in Theory of Inventive Problem Solving methodology (aka. TIPS). TIPS (Theory of Inventive Problem Solving) that is also called TRIZ (Teoriya Resheniya Izobretatelskikh Zadatch) is a methodology and model-based technology for generating innovative ideas and solutions for problem solving [9]. It has been developed by Genrich Saulovich Altshuller who have believed that learning how to invent is new things $[1,7,9]$ After his first approach, TRIZ has been evolved as the science of innovation and many companies are adopting TRIZ to solve complex technical problems.

TRIZ methodology is applied for user experiences enhancement in portable consumer electronics based on human behavior but simpler than a current innovative experience that is applied on i-phone by Apple or WII by Nintendo. The LBS application for low tier devices is the target for the improvement. TRIZ adaptation in mobile industries has been proposed by author [2] and this research is another tactical TRIZ approach to demonstrate the guidelines for applying into information technologies. Several TRIZ tools are applied for the project and the roadmap for problem solving needs to be indicated. The selected TRIZ tools are used for solving the problem in this resarch. The roadmap of TRIZ for a project is very important and the paper provide three steps of the problem solving for Location Based Service in mobile industry (see the Fig.1.)

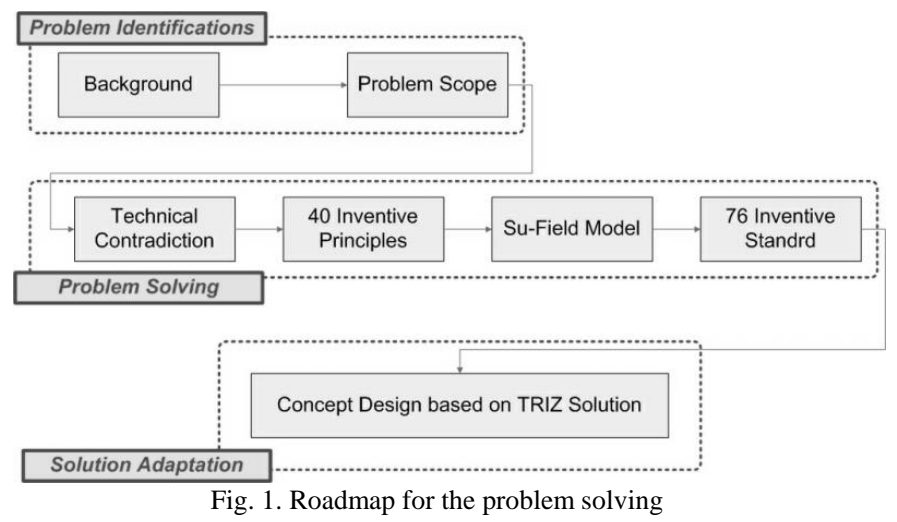

\section{TECHNICAL CONTRADICTION}

The sets of 40 inventive principles and 39 system features that one typically wants to be improved are defined in TRIZ. The system that has the technical contradiction can be clarified based on the feature for improvement [9]. Originally, Altshuller reviewed patents in order to find out what kind of contradictions were resolved or dissolved by benchmark the patents that have been achieved.

Location Based Service (LBS) is an information and 
entertainment service, accessible with mobile devices through the mobile network and utilizing the ability to make use of the geographical position of the mobile device by using Global Positioning System (GPS) [11]. GPS is a mandatory technology for LBS applications but it takes more than ten minutes to find the initial location position of a device. Assisted GPS (A-GPS) is design for gathering the initial position much faster but A-GPS is required higher application chipset process power. Currently, a LBS application is very common and it is embedded even in a low tier devices. The initial GPS position must be calculated before launching the LBS applications but required the additional process power.

Based on the above condition, the related technical contradictions (TC) are:

TC-1 : if the initial GPS position is calculated, it is easy to get the current position but not good for the device performance

TC-2 : if the initial GPS position is NOT calculated, it is hard to get the current position but good for the device performance

TC-2 is taken to design the enhanced LBS user experiences (see Fig.1b). Idle Final Result (IFR) can be defined as good for the initial GPS tracking without any performance reductions.

Based on the above conditions, Invention principle 10 (Preliminary action) and Invention principle 11 (Beforehand Compensation) are applied to solve the technical contradiction.

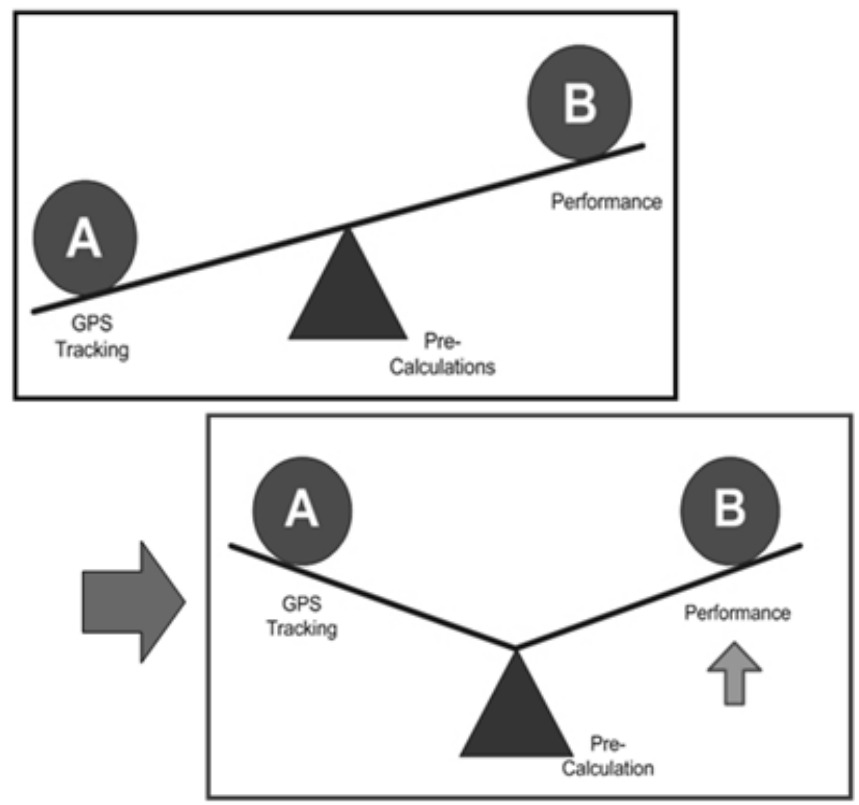

Fig. 1b. Idle Final Result of TC-2

\section{SubSTANCE-FIELD MODEL}

Genrich Altshuller and his colleagues identified 76 standard solutions to fixing problematic Su-Field models based on their intensive research of a huge number of patents $[1,3,6]$. Substance-Field (Su-Field) model is for analyze the problems related existing technological systems [6]. Su-Field model analysis is applied for making innovative problems to the form of analysis model related to the current technical system. It means that Su-Field model is the tool for identifying problems in a technical system and finding innovative solutions to these identified problems [3]. Based on Su-Field model, standard solution 1-1-3 is suitable for calculating the initial location position of the device without extra resources (see Figure 2.)

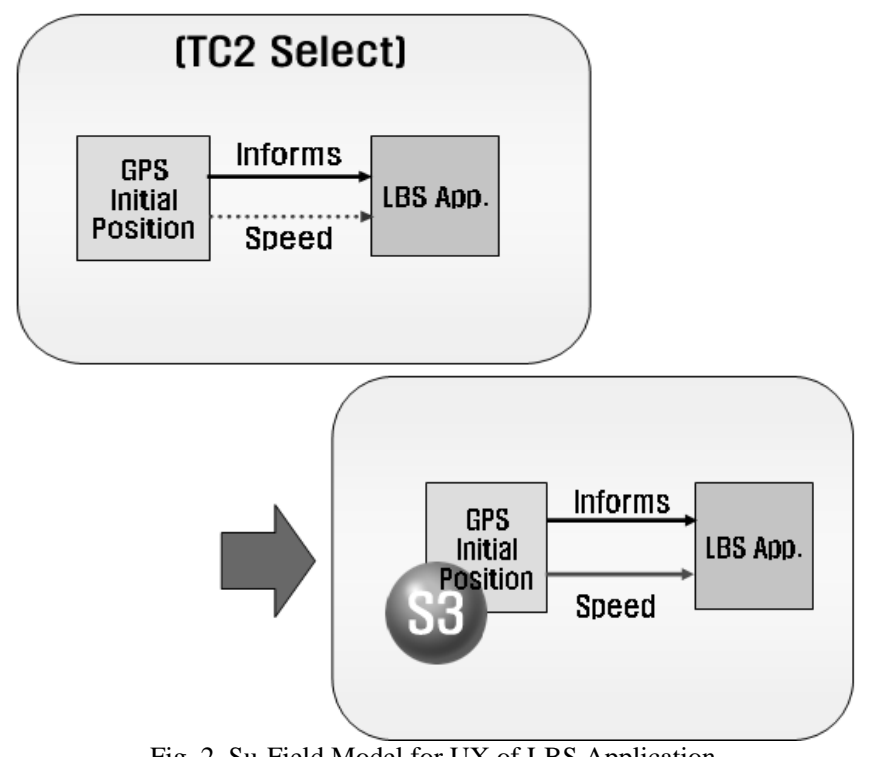

Fig. 2. Su-Field Model for UX of LBS Application

\section{DESIGN OF ENHANCED USER INTERFACES}

As the previous session mentioned, the enhanced concept is based on calculating the initial location more efficient way. Central Limit Theorem [8] is the main reason that makes the basic data within usual operations representing conventional human behavior. The statistical data are considered as the factors based on human behaviors. These data are very common from most of LBS applications. The cities that a user has been visited, the frequently used satellite are typical data that can show the related behaviors of a user. The following data must be collected for the enhanced user interfaces:

$$
\begin{gathered}
\quad L_{k}:=\text { The cities that have been visited } \\
C_{k}^{:}:=\text {Frequently visited cities }
\end{gathered}
$$

The next step is finding the values that are basic elements for the user experience enhancement for calculating the initial locations. Let $\mathrm{L}$ be the set of the frequently visited cities and $\mathrm{F}^{*}$ be sorted set of the frequently visited cities and as follow:

$$
\begin{array}{r}
\mathbb{L}=\left\{L_{1}, L_{2}, \ldots, L_{N}\right\} \\
\mathbb{F}^{\mathbf{2}}=\left\{C_{1}^{\mathbf{2}}, C_{2}^{\mathbf{2}}, \ldots, C_{N}^{\mathbf{2}}\right\}
\end{array}
$$

where $N$ is total number of the cities that the user has been visited. Each city has the index $(1, \ldots, N)$ that can identify the city be marked based on timely manner. The number of visiting for each city is also defined as the set $\mathbf{N}$ :

$$
\mathbb{N}:=\left\{n_{1}^{2}, n_{2}^{2}, \ldots n_{N}^{2}\right\}
$$

For instant, the first element in $\mathbf{F}^{*}$ is is the most visited city for the user and the first element in $\mathbf{N}$ is the number of times for visiting. Let $\mathrm{x}_{\mathrm{i}}, \mathrm{y}_{\mathrm{i}}$ be the horizontal and vertical locations of each city. The main value of the locations for each city can 
be found as the average of the locations for each city:

$$
\begin{aligned}
& \bar{x}_{i}^{2}=\frac{1}{n_{i}^{2}} \cdot \sum_{k=1}^{N} \mathbb{I}_{\left\{c_{i}^{*}-L_{k}\right\}} \cdot x_{k} \\
& \bar{y}_{i}^{2}=\frac{1}{n_{i}^{2}} \cdot \sum_{k=1}^{N} \mathbb{I}_{\left\{C_{i}^{*}-L_{k}\right\}} \cdot y_{k}
\end{aligned}
$$

The information of a satellite indicator is mandatory data for finding the initial location faster. The set of most frequent satellite indicators on the city as follow:

$$
\mathbb{G}^{\mathbf{2}}=\left\{G_{C_{1}^{+}}^{\mathbf{2}}, G_{C_{2}^{+}, \ldots, G_{C_{N}^{*}}^{\mathbf{2}}}^{\mathbf{2}}\right\}
$$

$\mathbf{F}^{*}$ and $\mathbf{G}^{*}$ are not only the sets as mathematical perspective but also actual city list and the satellite indicator list that contain the ordered name of cities and the indicators. Based on the set of the frequently visited cities, the workflows for the implementation as follow (see Fig. 3):

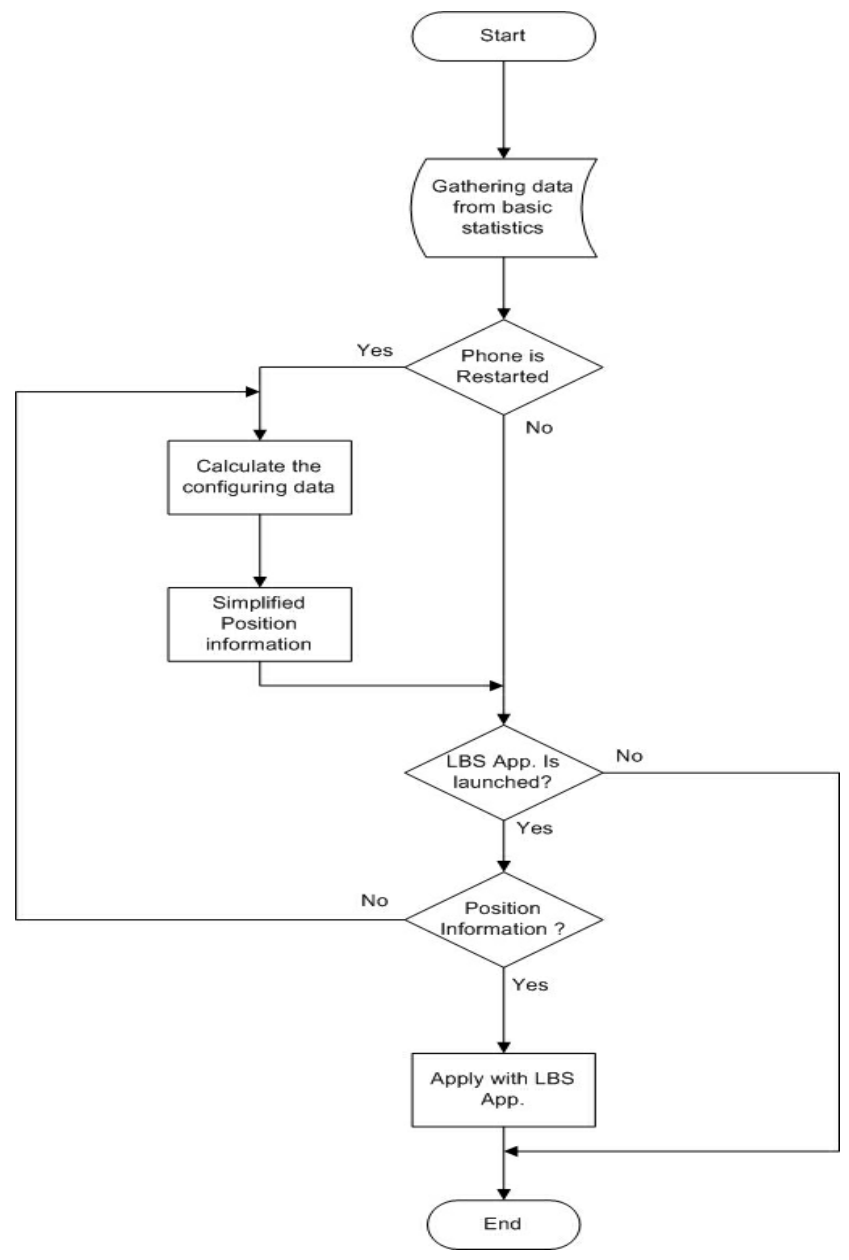

Fig 3. Workflow for enhancing the initial position

\section{CONCLUSION}

This research is targeting for developing the enhanced user experience design for location based services to give user the easy way for operating the devices. The faster positioning for the initial locations for LBS applications has been targeted as case study and the human behavior based approach can be adopted other area. Theory of Inventive Problem Solving (TRIZ) methodology makes possible to design the new types of user experiences implementations. Even though the research is dedicated with mobile industries, the pattern of TRIZ approach in the paper can be also applied to other industries.

\section{ACKNOWLEDGMENT}

The paper is the expansion for the proceedings of ICMSS (2011 International Conference on Management and Service Science) and submitted to IJSSH (International Journal of Social Science and Humanity) as the accepted publication under the agreement between ICMSS an IJSSH.

\section{REFERENCES}

[1] Altshuller, G., And Suddenly the Inventor Appeared: TRIZ, the Theory of Inventive Problem Solving, Technical Innovation Center, Worecester, MA (1996)

[2] Kim, S.-K., "Design of Event Driven DRM", IEEE Proceedings of $\operatorname{IEEM}$ (2007), pp935-938

[3] Mao, X. and et. al., "Generalized Solutions for Su-Field Analysis", The TRIZ Journal, August (2007)

[4] Polderman, J.W. and Willems, J. C., Introduction to Mathematical Systems Theory: A Behavioral Approach, Springer, New York, NY (1998)

[5] Thaler, R. H. and Simstein, C. R., NUDGE: Improving Decision About Health, Wealth and Happiness, Penguin Books, New York, NY (2008)

[6] Terninko, J., "Su-Field Analysis", The TRIZ Journal, February (2000)

[7] Terninko, J., Zusman, A. and et. al., Systematic Innovation: An Introduction to Theory of Inventing Problem Solving, CRC Press, Boca Raton, FL (1998)

[8] Trotter, H. F., “An elementary proof of the central limit theorem”, Arch. Math. 10 (1959), 226-234

[9] Rantanen, K.and Domb, E., Simplified TRIZ 1st ed., CRC Press, Boca Raton, FL (2002)

[10] Vincente and Kim, J., The Human Factor: Revolutionizing the Way People Live with Technology, Routledge, New York, NY (2006)

[11] Wang, S., Min, J and Yi, B. K., "Location Based Services for Mobiles Technologies and Standards", Proceedings of ICC (2008)

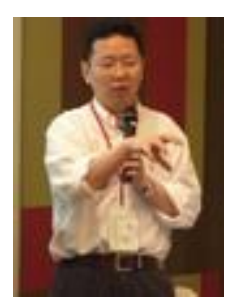

Dr. Song-Kyoo Kim is a Technical manager and certified 6 Sigma Black Belt (SSBB) and TRIZ specialist of mobile communication division at Samsung Electronics. Dr Kim has been doing stochastic and queuing modelling for 10 years. Dr Kim has received his master degree of computer engineering on 1999 and $\mathrm{Ph} . \mathrm{D}$. of operations research on 2002 from Florida Institute of Technology and also holds bachelor degree in Physics. He is the author of more than 20 operations research papers focused on queueing modeling, patents in mobile industries and the project leader of several 6 Sigma and TRIZ projects mainly focused on the mobile industry. 